\title{
Pigmented Skin Lesion Diagnosis by Automated Imaging System
}

\section{Mariam Ahmed Sheha ${ }^{1}$, Mai S Mabrouk ${ }^{2 *}$ and Amr Sharawy ${ }^{1}$}

${ }^{1}$ Biomedical and system Engineering, Cario University, Giza, Egypt

${ }^{2}$ Biomedical Engineering Department, MUST University, Giza, Egypt

\begin{abstract}
Pigmented skin lesions are the normal part of the skin, however its anomalous appearance is an annoying sign due to the presence of melanomas one of its malignant forms. Although melanoma is a deadly considerable disease, its early detection is a serious step toward mortality reduction. The proposed research discusses the outcome of introducing312 different features in a non-invasive diagnosis method for pigmented skin lesion diagnosis. To obviate the problem of qualitative interpretation, two different image sets are utilized to examine the proposed system, a set of images acquired by standard camera (clinical images) and another set of dermoscopic images captured from the magnified dermoscope. System contribution appears in using large conclusive set of features fed to different classifiers composing totally complete, new and different approaches for the purpose of disease diagnosis. Miscellaneous types of features used such as geometric, chromatic, and texture features extracted from the region of interest resulted from segmentation process. Then, the most prominent features that can cause an effect are selected by three different methods; Fisher score method, t-test, and F-test. The high-ranking features are used for the diagnosis of the two lesion groups using Artificial Neural Network (ANN), K-Nearest Neighbor (KNN) and Support Vector Machine (SVM) as three different classifiers proposed. System performance was measured in regards Specificity, Sensitivity and Accuracy. The ANN designed with the feature selected according to fisher score method enables a diagnostic accuracy of $96.25 \%$ and $97 \%$ for dermoscopic and clinical images respectively.
\end{abstract}

Keywords: Component; Pigmented skin lesions; Color space; Bounding box; SVM; Local range; KNN; ANN; Experimental models

\section{Introduction and Literature Review}

Skin cancer patients seeking for dermatologic consultation is steadily increasing worldwide. Melanoma often has a slow early growth rate the time lesions may be detected and removed at a relatively low cost and hazard. This resulted to raise survival rate to $95 \%$. While, progression of the disease in the late stage is associated with poor survival rateof $13 \%$ [1]. For that reason, melanoma detection weighted as a challenging problem for dermatologist. High expectations are entertained for inspecting lesions using technique named dermoscopy or Epiluminescence (ELM) [2]. ELM is an in-vivo, noninvasive technique used to enhance visualization of structures under the skin surface by means of oil immersion and magnifying instrument, called a "dermatoscope" [3]. However, dermoscopic images show a magnified view for skin lesions its interpretation depends mainly on the experience of the viewer. Several diagnosis models with similar reliability have become more widely accepted by physicians as, 7-point check, Menzie rule and the most popular scoring system so called ABCD rule [4,5].

Extract feature that compensate points in those systems is the error-prone operation during diagnosis. So that, automated diagnosis systems introduced as a non-invasive support tool to enhance the diagnostic capability of dermatologist if it is used as a second opinion. There are a considerable number of published researches in the area of image analysis and pattern classification related to the diagnosis of melanoma images. These diagnostic systems have developed over the last 20 years to have diagnosis accuracy around $73 \%$ to $98 \%$ [6].

July 2002, P Rubegni et al. [7] developed an automated melanoma recognition system with a set of 50 objective features subdivided into three categories (geometries, textures and islands of color) to describe the malignancy of a lesion. The13 significant features selected, are sufficient to discriminate the 2 groups of lesions reaching $94 \%$ accuracy using an ANN as the classifier. In 2006, Iris Cheng designed an algorithm using relative color features; the algorithm was tested upon 80 nevus, 160 melanoma and 42 dysplastic nevi, as a database. The result showed that the algorithm is able to classify $86 \%$ of the malignant melanomas successfully compared to an approximately $75 \%$ success rate of dermatologists [8].

February 2009, José FernándezAlcón, CalinaCiuhu built up an automatic system for diagnosis of pigmented skin lesions and melanoma that supports skin lesions images acquired using a conventional (consumer level) digital camera [9]. More importantly, that system includes a decision support component that used as a method of combination for the image outcome classification with context knowledge such as skin type, age, gender, and affected body part. The system classified images with an accuracy of $86 \%$, a sensitivity of $94 \%$, and specificity of $68 \%$.

Toward contributing information about the essential characteristics of pigmented skin lesion, in March 2012, Mariam A Sheha, Mai S Mabrouk, AmrSharawy proposed a Computer aided diagnosis system $(\mathrm{CAD})$ that mainly concerned by analyzing only dermoscopic images based on gray level Co-occurrence matrix (GLCM) and Using Multilayer perceptron classifier (MLP) to classify between Melanocytic Nevi and Malignant melanoma[10]. Moreover it was interesting to study the effect of this system using clinical images and compare results with dermoscopic images that was clearly represented on 2013 [11]. Other different features and classifiers were widely used in all fields of automated analysis and diagnosis that was valuable to introduce and study its effect. On Dec 2014, the effect of morphological features

*Corresponding author: Mai S. Mabrouk, Biomedical Engineering Department, MUST University, Giza, Egypt, Tel:+20 2 35676105; E-mail:msm_eng@yahoo.com

Received: September 15, 2015; Accepted: October 16, 2015; Published: November 30, 2015

Citation: Sheha MA, Mabrouk MS, Sharawy Amr (2015) Pigmented Skin Lesion Diagnosis by Automated Imaging System. J Bioengineer \& Biomedical Sci 5: 170. doi:10.4172/2155-9538.1000170

Copyright: @ 2015 Sheha MA, et al. This is an open-access article distributed under the terms of the Creative Commons Attribution License, which permits unrestricted use, distribution, and reproduction in any medium, provided the original author and source are credited. 
(Geometric and Chromatic) was handled using two different classifiers ANN and SVM [12].

This paper works as a completion for all the previous work. Whereas, its main aim is to design a fully automated computer aided diagnosis system of melanoma skin cancer to increase system flexibility and efficiency. It concerns about different types and a huge number of features to measure the effect of each upon melanoma diagnosis. Feature extraction is mainly based on two different concepts, namely morphological (geometric, chromatic) and texture analysis. The most prominent features describing melanoma were then selected by three different methods of feature selection f-test, t-test and fisher score ranking. Three classifiers were proposed to discriminate between malignant melanoma and benign nevi regarding the parameters selected.

The following sections introduce the digital image analysis system designed using different analytical parameters and classification methods performing different approaches. The results of these different approaches were compared to each other to investigate which is more suitable for a complete method for melanoma diagnosis.

\section{Materials}

\section{Database}

Toward productive screening process various non-invasive, in-vivo imaging technique proposed. The imaging modalities used nowadays could also allow the visualization of skin lesion structure such as, Positron Emission Tomography (PET), Dermoscopy, Confocal laser scanning microscopy (CLSM), High frequency ultrasound, Optical coherence tomography (OCT) and Magnetic Resonance Imaging (MRI) [13]. The study proposed applied upon a set of traditional photography, which was used for a long time in dermatology named by clinical images and another set of dermoscopic images [14,15].

The main aim through this research is to define the effective characteristics for designing a high performance consumer application CAD system with high prediction accuracy. So that, it was recommended to apply the same algorithm on both types of data sets mentioned below to measure the effect of different imaging techniques upon the diagnosis.

Through this research, 320 lesion images of different cases were randomly sampled from the skin diseases atlases and doctors' clinics. For each dermoscopic and clinical images 160 lesions were used. Images were divided equally between benign nevi and malignant melanoma, to have 80 lesions for each group. Image atlases of dermoscopic images are widely available in $[16,17]$. The majority of the dermoscopic images were randomly sampled from [18] as the author of [19] recommends them as a melanoma database. The clinical images used for that purpose are widely available in [20-24]. All images are fairly divided for two classes $50 \%$ for learning set and 50\% for test set.

\section{Implementation}

For successful diagnosis and high performance automated system a good visualization is essential. Leveraging the rich graphics functionality of MATLAB, the proposed system is implemented and tested on the WINDOWS platform with MATLAB version of 2010.

\section{Methodology}

The objective of this research is to apply different and new features on a computer-based system forming different experimental approaches. For that purpose it is valuable to detect the region of interest so the PSL can be accurately evaluated. Once the lesion boundary had been identified, the program attempts to extract different features to manipulate their importance in that field. After wards, classified to automatically assess the accuracy degree of that approach. The features used with the most accurate approach will be recommended to recognizes dermoscopic findings as accurately as expert dermatologists. Figure 1, outlines the procedure followed by the different approaches used to diagnose PSL for both types of database mentioned above.

\section{Image Segmentation}

Dermoscopic and clinical images are often altered by uneven illumination, and the same goes to typical skin artifact as hair, skin structures, oil bubbles [25]. Artifact affects directly both segmentation and feature extraction algorithms. Image segmentation is an essential preprocessing step that copes with skin irregularities, artifacts and with a curved surface. Sequentially, it is necessary for efficient feature extraction and accurate classification. So that, three pre steps of processing were considered, which is image preparation, image enhancement then thresholding.

\section{Image preparation}

That step goes through adjusting the image size to $470 \times 640$ pixelsdue to the wide size variation found in different scaled images.

\section{Images enhancement}

Segmentation can be distracted by the presence of skin artifacts. Two successive steps are necessery to improve the visibility of the tumor border and ensure that it is correctly identified, filtration then conversion to intensity image.

a) Filteration: The median filter calculates the median value of the intensity in the pixels encompassed in the filter area and replaces the intensity in the center pixel with this value [26]. So, it is a correction tool for reducing noise, which has corrupted the clarity of the image. Although this procedure ends in relatively blurring of the image as shown in Figure 2, it assists the segmentation procedure.

b) Gray scale conversion: To reduce the effect of different skin color variations and background, the RGB colored image is converted into intensity or gray scale image [27].

\section{Images thresholding}

In general segmentation is the process of grouping pixels of a given image into regions, which are homogenous with respect to certain features and to semantic content. The purpose is to extract the lesion border from the healthy skin, subdividing an image into meaningful non-overlapping regions, it would be used for further analysis and background representation of the healthy skin [28]. Contrast adjustment should precedeimage thrsholding process where it always play an effective role at detecting the correct boundary.

a) Contrast Adjustment:Image is adjustedby saturating $1 \%$ of data at low and high intensities of output image. Figure 3 shows the effect of adjustment process upon the filtered intensity image.

b) Image Threshold: It mainly depends on Otsu's method, where it labels each pixel of the entire image as belonging to the lesion or to the background. It searches for the threshold value that the intra-class variance minimizes, defined as a weighted sum of variances of the two classes [29]. Threshold is used to extract an object from its background 


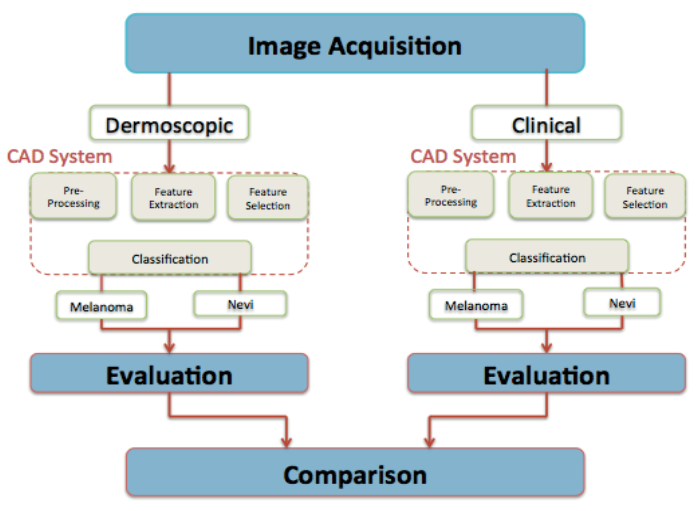

Figure1: Main block diagram.

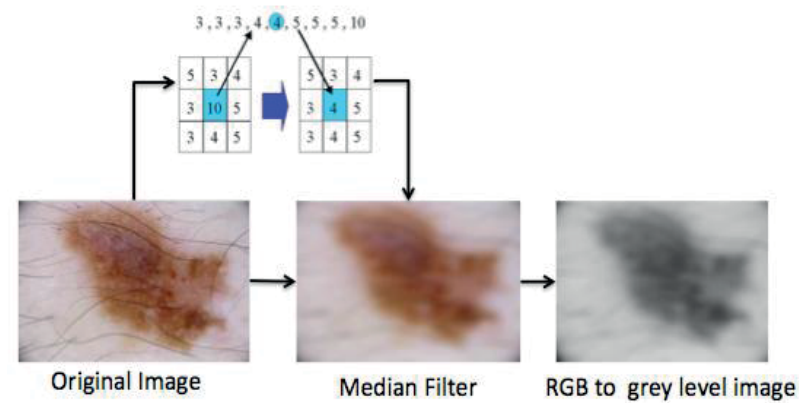

Figure 2: Image Enhancement process.

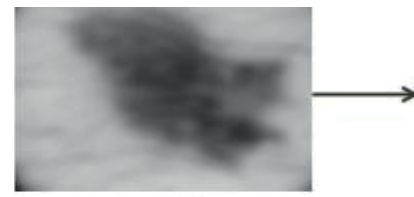

Gray Level Image
Intensity adjusted

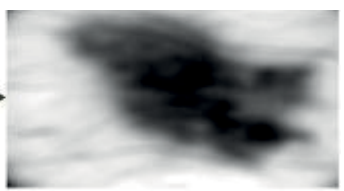

Figure 3: Image contrast adjustment.

by assigning an intensity value $\mathrm{T}$ (threshold) for each pixel such that each pixel is either classified as an object point or a background point. Individual pixels in an image are marked as "object" pixels if their values are less than the threshold value and as "background" pixels otherwise. Figure 4 shows the conversion happened as a result of Otsu's threshold.

After the threshold process had been accomplished, it is supposed that segmentation process is almost finished, but important three fine steps still remains, namely to have accurate tumor detection without being confused with any other background disorders. To clear out the background from any undesirable objects, any object with size less than 150 pixels were removed by means of morphological operations [30]. Then, Binary image is inverted to refer for the selected object by white pixels "ones" and black pixels "zero" for background. Also, morphological operations were then used to fill in gaps that may found at lesion mask to complete tumor holes. Finally, depending on the lesion contour detected regarding the traced boundary [31], lesion center of mass (centroid) and bounding box which is the smallest rectangle containing the region were defined. Figure 5 Shows an example for complete segmentation process [7].

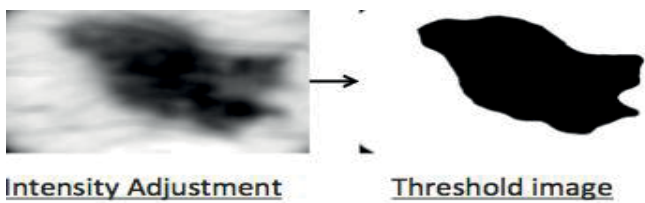

Figure 4: Threshold process.

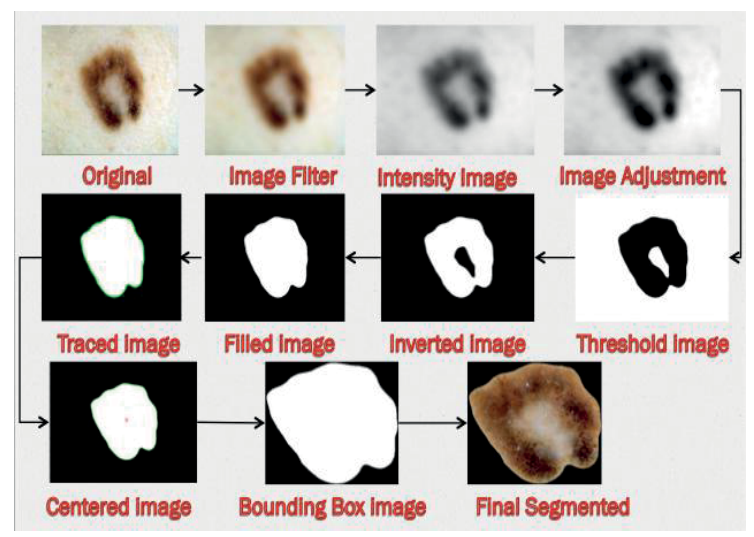

Figure 5: Schematic representation for image Segmentation steps.

\section{Feature Extraction}

Segmentation operated and reduced number of objects in the pigmented lesion to concern with the required region. Then variety of feature sets representing the essential lesion characteristics need to be extracted in order to decide whether the lesion is benign or malignant. The features are such that value ranges can be assigned within which the diagnosis can be made specific with high probability.

The most valuable prognostic factor of malignant melanoma is appearance variability. It is irregular in shape and color with differential structure. On this spot, that research attempt to compromise that heterogeneous phenomena proposing and examining 312 different features. The features presented study lesion property from three concerned sides geometric for shape, chromatic for color and texture features representing differential structure.

\section{Geometric features}

Shape descriptors supply important information to discriminate malignant melanoma from melanocytic nevi. Those features are efficient at performing shape analysis of objects in a binary image. Results show the effectiveness of the geometric features. Measuring a set of properties for the segmented binary image, 21 geometric featureswere computed $[9,30]$. By looking at the schematic region in Figure 6. geometric features are described as follow. Area $\left(A_{p}\right)$, Perimeter $\left(P_{p}\right)$, Center of gravity (Centroid), Solidity $\left(\mathrm{A}_{\mathrm{p}} / \mathrm{A}_{\mathrm{c}}\right)$, Roundness, Irregularity, Major axis $\left(a_{p}\right)$, Minor axis $\left(b_{p}\right)$, Ratio of axis $\left(a_{p} / b_{p}\right)$, Eccentricity, Extent $\left(A_{p} /\right.$ $\left.A_{b}\right)$, Equivalent Diameter, Radius, Bounding Box area $\left(A_{b}\right), x$-axis of bounding box $\left(b_{b}\right), y$-axis of bounding box $\left(a_{b}\right)$, ratio of bounding box $\operatorname{axis}\left(\mathrm{b}_{\mathrm{b}} / \mathrm{a}_{\mathrm{b}}\right)$, Estimated borderline, Convex Hull, Convex Hull area (Ac), Orientation. 


\section{Chromatic features}

Features such as color, brightness or luminance, texture and shape could be aguide to distinguish skin cancer from the surrounding skin. Skin color has proven to be a useful and robust cue for diagnosing malignant melanoma [32]. Skin lesion could be examined by different color spaces rather than RGB. As color space is a mathematical representation of a set of colors, various color spaces exist. They present color information in ways that make certain calculations more convenient or because they provide a way to identify colors that is more intuitive. The seven color spaces introduced through that study and different feature vector extracted out of each are brefily definedthrough this section. (Figure 6)

The RGB is the true color space that defines a color as the percentages of red, green, and blue hues additive colors mixed together. Luminance and two color difference signals represented by YIQ and $\mathrm{YCbCr}$ represented important color spaces especially in the field of video standard. The Y component represents the luminance information, it is the only component used by black-and-white television receivers where $\mathrm{I}, \mathrm{C}_{\mathrm{b}}$ and $\mathrm{Q}, \mathrm{C}_{\mathrm{r}}$ represent the chrominance information [33]. However, none of these color spaces directly related to the intuitive notions of hue, saturation, and brightness. This has resulted in the pursuit of other models, such as HSI and HSV to simplify processing and identify more details and information about the lesion [34]. Also, $L^{*} u^{*} v^{*}$ and lab spaces are valuable color spaces previously mentioned in different studies were introduced [35]. Regarding the color spaces and the different effect occurring on the lesion by each, it is important to investigate features that represent that change. Three-fixed, valuable, and easy to extract feature are extracted from each component of the color spaces. They are specifically the mean, standard deviation and the ratio between them, which named by 'coefficient of variation' and finally sum of color space component. a) Mean $(\mu)$ : It is calculated as the average of the intensity pixels belonging to the lesion. b) Standard Deviation $(\sigma)$ : This value was taken as the color variation risk. In general, lesions with a higher color standard deviation are considered to be a higher cancer risk. It was calculated as the standard deviation of the grayscale intensity values of all the pixels belonging to lesion regions. c) Coefficient of variation: It can be seen as the relative standard deviation that can be calculated by dividing the standard deviation with the mean value. d) Sum of color component:Another new chromatic feature was proposed that considered the sum of the intensity values over the three channels of the color space, i. e for the true space the feature represented by the intensity sum of red, green and blue, as $S=R+G+B$ Accordingly, 70 chromatic features computed to provide more valuable and somehow complete color information for correct malignant melanoma diagnosis. The seven color spaces represented in Figure7 were analyzed and used to finally consider 10 different chromatic features out of each.

\section{Texture features}

Most of the tumor is heterogeneous tissues and the values represented geometric and chromatic features are not at all sufficient to characterize the heterogeneity of the different tumor types, where it can describe melanoma different structures. The intensity of the color as well as thepattern of the pigmentation - so called texture -are also very importantfeatures for the detection of melanoma. Since different lightening conditions change the apparent texture of a lesion [36], different features were computed for each of the seven color spaces proposed to circumvent the problem. Textures represent tonal variations in the spatial domain and determine the overall visual smoothness or coarseness of image features.

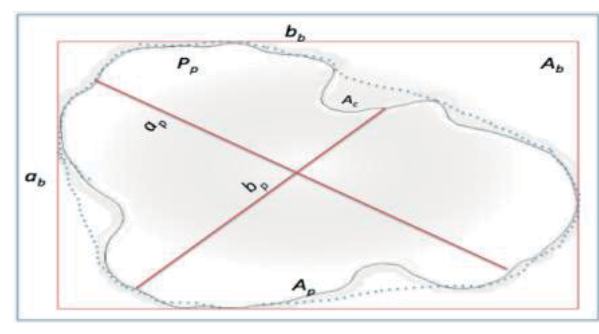

Figure 6: Parameters of the bounding box containing segmented area.

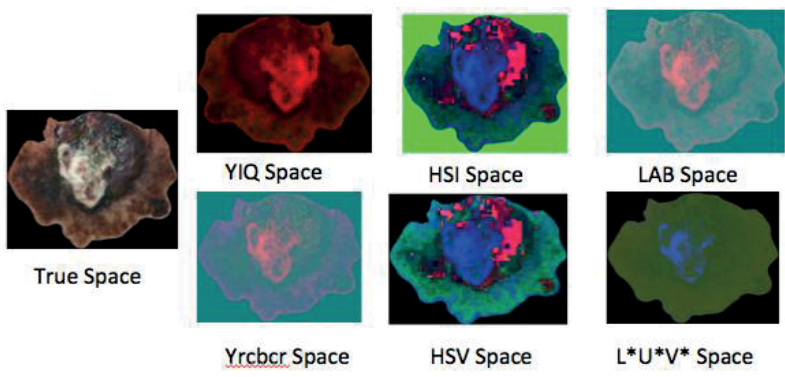

Figure 7: Malignant melanoma lesion represented by the 7 color spaces.

Important features about skin texture were extracted by evaluating lesion entropy of the image on the different color spaces: RGB, HSV, HSI, YCbCr, YIQ, LAB, $L^{*} u^{*} v^{*}$ to obtain 7 different features. Furthermore, texture analysis includes several functions that filter an image using standard statistical measures such as range, standard deviation, and entropy [37]. These statistics can characterize the texture of an image because they provide information about the local variability of the intensity values of pixels in an image. For example, in areas with smooth texture, the range of values in the neighborhood around a pixel will be a small value; in areas of rough texture, the range will be larger. Similarly, calculating the standard deviation of pixels in a neighborhood can indicate the degree of variability of pixel values in that region. Accordingly, the means and standard deviations of local entropy, local standard deviation and local range of gray scale image are added as separate features for each color component of the seven spaces counting out one hundred and twenty- six features that could be defined as follow. a) Local entropy:Local entropy of image is the array obtained where each output pixel contains the entropy value of the 9-by9 neighborhoods around the corresponding pixel in the input image [37]. b) Local standard deviation: Itoperates similarly to local entropy, defining a neighborhood around the pixel of interest and calculating the statistic for the neighborhood to determine the pixel value in the output image. It is the array obtained where each output pixel contains the standard deviation of the 3-by-3 neighborhoods around the corresponding pixel in the input image [38]. c) Local range:It attempts to quantify intuitive qualities described by terms such as rough, silky, or bumpy in the context of an image. In this case, the roughness or bumpiness refers to variations in the brightness values or image gray levels. Local range of an image is the array obtained where output pixel contains the range value between maximum value and minimum value of the 3-by-3 neighborhoods around the corresponding pixel in the 
input image [38].

Further information about skin texture can also be mentioned by extracting the Gray Level Co-occurrence Matrix (GLCM) features out of image transformed to the different color spaces used. The four GLCM features presented through that study are Energy, Homogeneity, Correlation and Contrast based on their classification accuracy, computational cost and previously usage in literatures [39]. Number of 88 features extracted according to the GLCM, where each of the four features extracted from each color component of the 7 color spaces $(7 \times 3 \times 4)$, in addition to, the four features extracted from the gray scale image of the true color space (RGB).

Number of texture features extracted considering the different seven color spaces presented was two hundred and twenty one features. A Simple representation of the distribution of the feature extracted based on different color spaces shown in Figure 8.

\section{Feature Selection}

Features extracted are not equally valuable for this classification task according to redundancy or irrelevancy. Additionally, data of a larger test set is hard to experimentally analyze and recognize patterns, and in turn will increase processing time, as some of these features are depend on somewhat heavy calculations. Therefore, various feature selection techniques and algorithms are used to reduce the number of features significantly by eliminating features with little or no predictive information. The three statistical methods proposed for the target of feature selection was, the F-test, the T-test and the Fisher score ranking to compare and increase the detection accuracy.

\section{F-Test}

It is the most commonly statistic measure used in Analysis of Variance (ANOVA); it can be regarded as a comparison of two variances. In other words, it is designed to test if two population variances are equal $[39,40]$. Accordingly, the f-value can be easily calculated using eq. 1.

$$
\mathrm{F}_{\text {value }}=\frac{\mathrm{S}_{1}^{2}}{\mathrm{~S}_{2}^{2}}
$$

Where: S1 and S2 is the Variance of first and second group respectively.

T-Test: It is the statistic measure that clears out if features are approximately similar in average to each other. It assesses whether the means of two groups are statistically different from each other relative to the spread or variability of their scores [41]. Statistic of t-test identified through eq. 2.

$$
\mathbf{t}=\frac{\bar{X}-\bar{Y}}{\sqrt{\frac{\mathbf{S}_{\mathbf{x}}^{2}}{\mathbf{n}}+\frac{\mathbf{S}_{\mathbf{y}}^{2}}{\mathbf{m}}}}
$$

Where: $\bar{X}$ and $\bar{Y}$ are the sample means, $\mathrm{s}_{\mathrm{x}}$ and $\mathrm{s}_{\mathrm{y}}$ are the sample standard deviations, and $\mathrm{n}$ and $\mathrm{m}$ are the sample sizes.

In statistical significant testing T-test and F-test, the p-value is the significant level of probability obtaining a test statistic at least as extreme as the one that was actually observed, assuming that the null hypothesis is true. Features with practically different variance was chosen as significant features where their p-values are suppose to be less than the significant level used for that study which is 0 . 05 . The smaller the p-value, the more significant is the difference between the distributions of the two populations, as shown by Figure 9 .

\section{Fisher score ranking}

It is a widely used feature selection methods. The idea of Fisher ranking is to find a subset of features, such that in the data space spanned by the selected features, the distances between data points in different classes are as large as possible, while the distances between data points in the same class are as small as possible. The algorithm calculates the difference between the malignant and benign lesions relative to a certain feature, in terms of mean and standard deviation, [42]. It seems to be the same rule used to evaluate the t-value at the t-test but the main difference appears in the way of choosing feature significance. Using the t-test features are considered significant, if their $\mathrm{p}$-value is less than a recommended alpha (0.05). For fisher ranking, the feature with the highest ranks were selected to minimize the computation time, following a ranking threshold for example of 0.5 .

\section{Classification}

Classification is defined as a process of finding a set of attributes to distinguish between classes of data for the purpose of predicting the class of objects, whose class label is unknown. Generally a classifier generates a decision boundary, which separates the points in feature space into two or more sets of classes. Each type of classifier will arrive at a decision boundary in a different way according to its working process and the classifier types have. The different classifying algorithms introduced to distinguish the classes of data are the Artificial Neural Network (ANN), the k-Nearest Neighbor classifier (K-NN) and Support Vector Machine (SVM).

This section concerned with discussing the main idea of the three

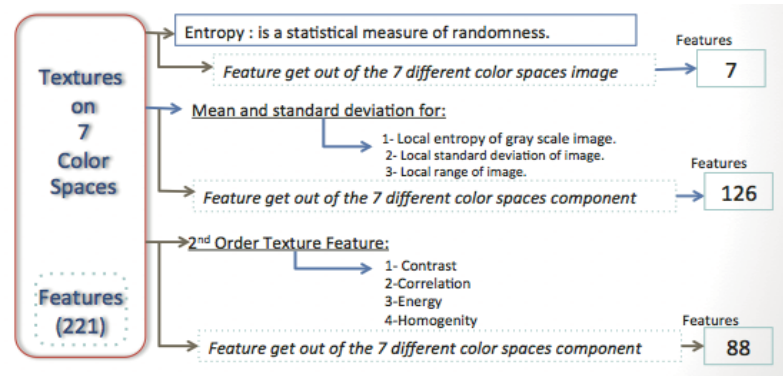

Figure 8: Texture features of 7- color spaces structure.

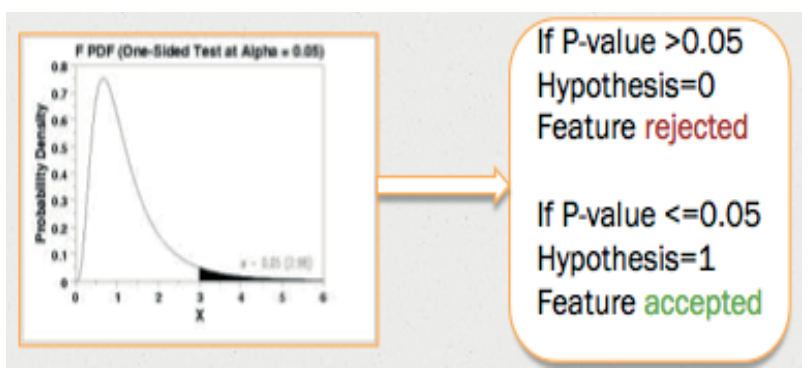

Figure 9: P-value graph. 
different classification models proposed to discriminate between malignant melanoma and benign lesion. The classification pattern was developed using half the database number for training and the other half for testing the classifier. There are 160 images for each data set whether dermoscopic or clinical image as previously mentioned in the material section. So; 80 images representing 40 for malignant melanoma cases and 40 of benign nevi cases used as a training set to accomplish the automated detection system of tumors, whereas the other 80 image having the same distribution for testing the automated detection system.

\section{Artificial Neural Network (ANN)}

The ANN's original aim was to understand and shape the functional characteristics and computational properties of the brain when it performs cognitive processes such as concept association, categorization and learning. For that reason, the ANN was the first choice of classification algorithm among all CAD software programmers [43].

To decide on a network topology some points must be considered for designing ANN classifier, Number of input nodes represented by the number of selected features feeded into the classifier. Number of output nodes is just one where it suppose to have a clear-cut if that image is whether malignant or benign. Then, Number of hidden nodes and Activation function are selectable according to the designed network and finally the network Training behavior. Accordingly two techniques were proposed for our study, the Automatic and Traditional ANN technique [12].

\section{K-Nearest Neighbor (KNN)}

$\mathrm{KNN}$ has recently been recognized as one of the best algorithms for clustering data as it is simple, quick and effective. $\mathrm{K}$ indicates the number of neighbors, then each sample, is classified in the same class, as the training data is closest [43]. It is training idea performed each neighbor of a sample to be classified is considered as an item of evidence that supports certain hypotheses regarding the class membership of that pattern. Finally the test image is classified as belonging to the class, which is most frequent among the $\mathrm{K}$-samples nearest to it.

\section{Support Vector Machine (SVM)}

It have shown excellent performance in many real-world applications including hand written digit, object recognition, speaker identification, face detection also at images and text categorization. SVM estimates the function that powerfully classifies data into two classes. It is based on the idea of minimizing the generalization error when the classifier is applied to test samples that do not exactly match any training sample used to train the classifier[44-49].

SVM main idea is that it classifies data by finding the best hyperplane that separates all data points of one class from those of the other class. The best hyper-plane for the SVM means the one with the largest margin between the two classes. The distance between two hyper-planes defines a margin and this margin is maximized when the norm of the weight vector is minimum. Figure10 illustrates SVM methodology of classification.

In order to obtain satisfactory predictive accuracy, different SVM kernel functions were proposed. Regarding that the main difference occurs between classifiers according to their training procedure. Three different SVM approaches have been proposed for classification as a result of using three different kernel functions that are linear, quadratic and RBF kernels [50-52].

\section{Evaluation}

To measure the performance level of the diagnosis done by the system, statistical measures called sensitivity and specificity calculated evaluating the diagnostic accuracy. These measures are used to describe the error rate by the terms true and false positive and true and false negative [53].

\section{Experimental results}

Among the previous sections the algorithms required for PSL diagnosis had been performed. Image was enhanced and segmented, different features had extracted out of that region of interest then the most promotes was selected. The resulted selected features worked as input for the three used classifiers to have multi different results according to each feature type, selection method and classification. This section intent to monitor the classification results and analyzes its performance among different features used. According to different features extracted and methods used for feature selection and classification there are too many experiments had performed. Measuring performance to like such huge number of experiments was handled by dividing experiments according to features category. Experimental approaches were divided into single and combined models; Figure 11 shows the description of the different approaches applied for both single and combined models.

\section{Single models}

According to the different types of features extracted, three single models are proposed. For that different experiments were operated,

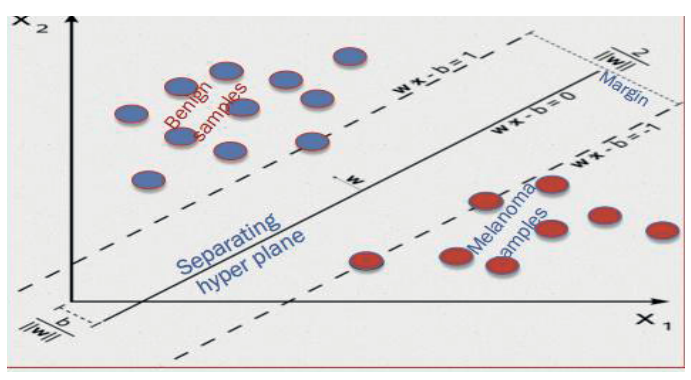

Figure 10: SVM Classification

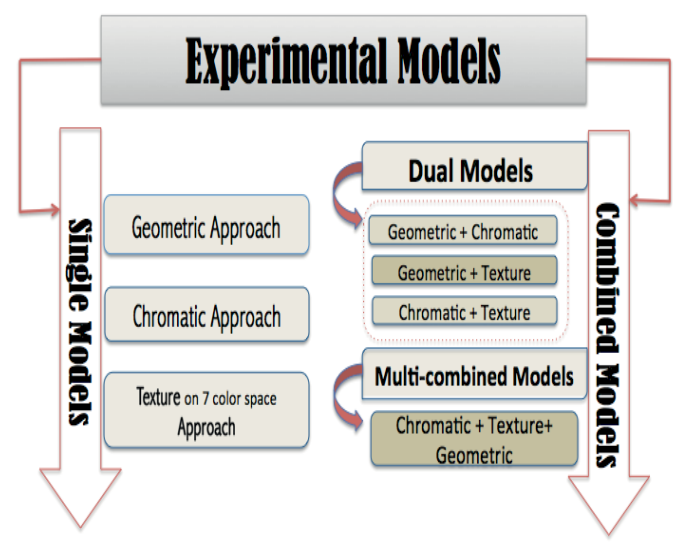

Figure 11: Single and Combined Model approaches. 
resulting from using 3 different sets of selected features fed into 6 different classifiers applied to the dermoscopic and clinical data set.

After the elimination of the least significant parameters, according to F-test, T-test and Fisher score ranking, it was noticed that the best accuracy of $93.75 \%$ occurred as a result of using 15 geometric features selected by fisher for both databases. Regarding chromatic features the classification results were promising for dermoscopic and clinical images, reaching $92.5 \%$ and $93.75 \%$ respectively classified by ANN. On the other hand, texture features were not very promising for digital images rather than dermoscopic lesions reaching accuracy of $86.25 \%$, $90 \%$, respectively when classified by linear SVM.

\section{Combined models}

Regarding the single models experimental results it was investigated that features selected by fisher score ranking method are the most significant ones, and so, were used for combined models structure. Accordingly, four combined approaches were proposed, three approaches were considered by combining two different feature types together dual models could name those. The fourth approach was proposed by combining the three feature types together (Geometric + Chromatic + Texture). Fifteen and twenty-two features represent geometric and chromatic features respectively for both the dermoscopic and clinical database. Forty- seven and twenty texture features selected to represent dermoscopic and clinical database respectively.

Dual models: Geometric features record the highest results when combined with texture or chromatic features for the two different types of databases. The best accuracy recorded was $95 \%$ for geometric and chromatic features sets of dermoscopic images classified by linear SVM. Where also the combination between geometric and texture feature of clinical images recod the same percentage when classified by automated ANN.

Multiple models: It was further observed that the amalgamation between the three different features category concluded a well representation for lesions whether of dermoscopic or clinical images. A number of eighty-four features chosen as the best features extracted out of dermoscopic images according to the three categories (Geometric+ Chromatic+ Texture) recorded accuracy of $96.25 \%$ when the feature set classified by traditional ANN. On the other hand clinical database represented by fifty-seven features recorded an accuracy of $97.5 \%$ when traditional ANN classified the feature set. Figure 12, 13 shows an accuracy chart for both dermoscopic and clinical images regarding the different combined models.

\section{Conclusion}

The non-invasive automated system for PSL and melanoma diagnosis supports two different types of skin lesions images. The system is designed considering 320 melanocytic skin lesions of both clinical and dermoscopic images. It starts with image segmentation to extract the infected lesion out of the healthy tissue. Then features extraction provides avaluable contribution; it defines the characteristics of thelesionimage by considering the description of the relevant properties of the image in a feature space. Features categories vary between91 morphological features based on (geometric, chromatic) and 221 representing texture analyses. Features efficiency were evaluated and selected by three different methods of feature selection f-test, t-test and fisher score ranking to look after the most significant ones. The selected features are feed into three different classifiers, Artificial Neural Network (ANN), K-Nearest Neighbor (KNN), Support Vector Machine (SVM). Results obtained concluded that, the

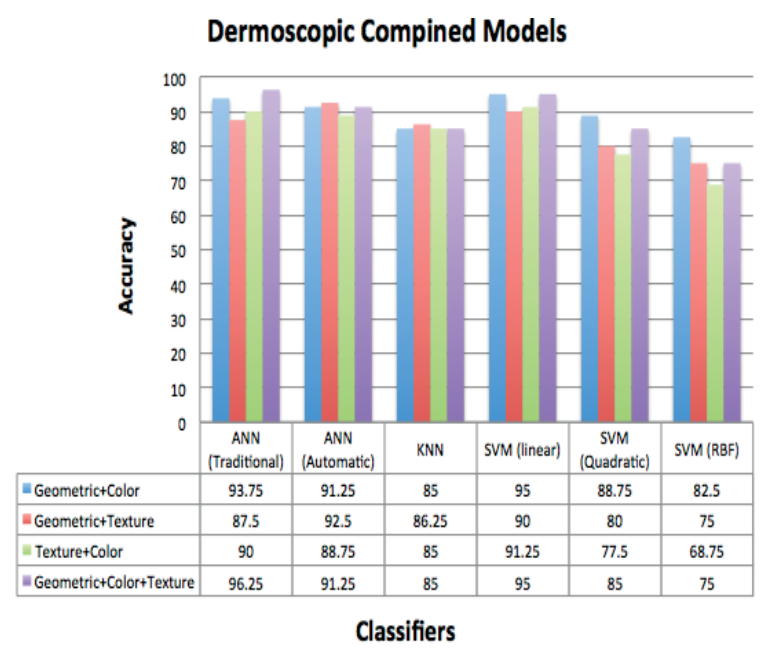

Figure 12: Dermoscopic combined models results.

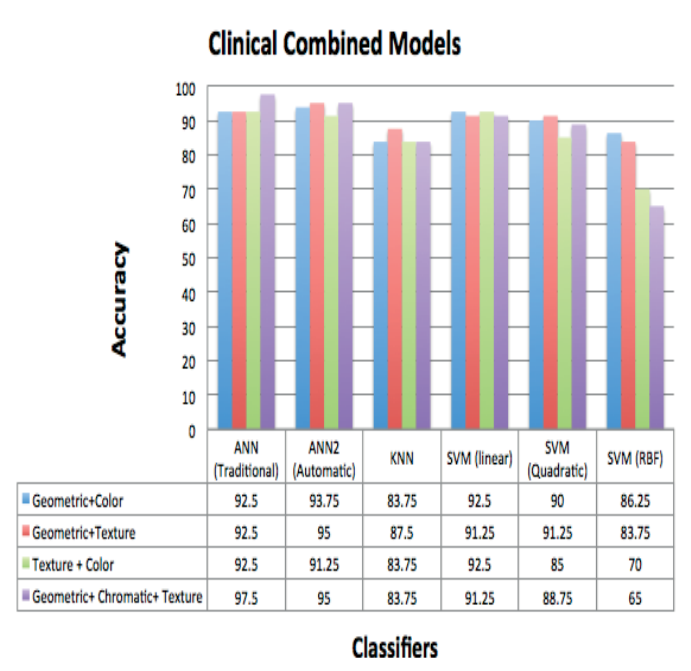

Figure 13: Clinical combined models results.

proposed study describes a software tool which implements different image processing algorithms developed for the automated analysis of clinical and dermoscopic images. More specifically, the use of different features categories as well the application of different classifiers, such as Machine Learning and Neural Network Techniques to pigmentation skin lesion images have been resulted in an integrated architecture for CAD. Experimentally it was shown that the co-operation between the three different features type geometric, chromatic and texture features selected regarding fisher score ranking had effectively defined the lesion structure rather than using only one or two feature types. Where the multiple model records 96.25 and $97.5 \%$ as the best accuracy resulted upon classifying dermoscopic and clinical images respectively by ANN. Comparing results achieved through the last multiple model to the recent studies mentioned in the literature review section, this approach considered as promising completed CAD system that add all different features category to cope with the lesion heterogeneous phenomena and diagnose it with accuracy reaches $97.5 \%$.

\section{References}

1. U.S. Emerging Melanoma Therapeutics Market (2001) Frost \& Sullivan Research Service. 
Citation: Sheha MA, Mabrouk MS, Sharawy Amr (2015) Pigmented Skin Lesion Diagnosis by Automated Imaging System. J Bioengineer \& Biomedical Sci 5: 170. doi:10.4172/2155- 9538.1000170

Page 8 of 8

2. Ascierto PA, Palmieri G, Celentano E, Parasole R, Caraco C, et al. (2000) Sensitivity and specificity of epiluminescence microscopy: Evaluation on a sample of 2731 excised cutaneous pigmented lesions. Br J Dermatol142:893-8.

3. Binder M, Chwarz M, Winkler A, Steiner A, Kaider A, et al. (1995) Epiluminescence microscopy: A useful tool for the diagnosis of pigmented skin lesion for formally trained dermatologists. Archives of Dermatology 131: 286291

4. Liu W, Hill D, Gibbs A, Tempany M, Howe C, et al. (2005) What features do patients notice that help to distinguish between benign pigmented lesions and melanomas?: The $\mathrm{ABCD}(\mathrm{E})$ rule versus the seven-point checklist. Melanoma Res 15: 549-554.

5. Nachbar F, Stolz W, Merkle T, Cognetta A B, Vogt T, et al. (1994) The ABCD rule of dermatoscopy: High prospective value in the diagnosis of doubtful melanocytic skin lesions. J AmerAcadDermatol30: 551-559.

6. Lau HT, Al-Jumaily A (2009) Automatically Early Detection of Skin Cancer: Study Based on Neural Network Classification. International Conference of Soft Computing and Pattern Recognition, Malacca.

7. Rubegni P, Cevenini G, Burronietalii M, Perotti R, Dell'Eva G, et al. (2002) Automated diagnosis of pigmented skin lesions. International Journal of Cancer 101: $576-580$.

8. Cheng I, Edwardsville, Illinois (2005) Skin tumor classification using relative color features. Thesis submitted in partial fulfillment for Master's degree in Electrical Engineering at Southern Illinois University.

9. FernándezAlcón J, Ciuhu C, Kate WT, Heinrich A, Uzunbajakava N et al. (2009) Automatic Imaging System With Decision Support for Inspection of Pigmented Skin Lesions and Melanoma Diagnosis. IEEE Journal of Selected Topics in Signal Processing: 3

10. Sheha MA, Mabrouk MS, Sharawy A (2012) Automatic Detection of Melanoma Skin Cancer using Texture Analysis. International Journal of Computer Applications 42: 22-26.

11. Mabrouk MS, Sheha MA, Sharawy AA (2013) Computer Aided Diagnosis of Melanoma Skin Cancer using Clinical Photographic images. International ournal of computer and technology 10.

12. Sheha MS, Mabrouk MS, Sharawy AA (2014) Pigmented Skin Lesion Diagnosis Using Geometric and Chromatic Features. Biomedical Engineering Conference (CIBEC), 2014 Cairo International, IEEE, Giza

13. Korotkov K, Garcia R (2012) Computerized analysis of pigmented skin lesions: A review. Artificial Intelligence in Medicine 56: 69-90.

14. Blum A1, Luedtke H, Ellwanger U, Schwabe R, Rassner G, et al. (2004) Digital image analysis for diagnosis of cutaneous melanoma. Development of a highly effective computer algorithm based on analysis of 837 melanocytic lesions. British Journal of Dermatology 151: 1029-1038.

15. Ratner D, Thomas C, Bickers D (1999) The uses of digital photography in dermatology. Journal of the American Academy of Dermatology 41: 749-56.

16. www.dermoscopic.blogspot.com/

17. www.dermoscopyatlas.com

18. http://www.ee.siue.edu/ sumbaug/Borders\%20Complete/

19. Zapirain BG, Zorrilla AM, Oleagordia IR, Nunez V, Abtane A (2009) Skin cancer parameterization algorithm based on epiluminiscence image processing.Signal Processing and Information Technology IEEE: 236 - 241

20. www.dermnet.com

21. Dermnetnz.org

22. www.dermQuest.com

23. Dermis.com (dermis.multimedica.de)

24. dermatlas.med.jhmi.edu

25. Lee T K (2001) Measuring border irregularity and shape of cutaneous melanocytic lesions. Simon Fraser University.

26. Jalil B, Marzani F (2008) Multispectral Image Processing Applied to Dermatology. A Thesis Submitted for the Degree of MSc Erasmus Mundus in Vision and Robotics (VIBOT).

27. Kumar T, Verma K (2010) A Theory Based on Conversion of RGB image to
Gray Image. International Journal of Computer Applications 7: 7-10.

28. Brake GM, Karssemeijer N (2001) Segmentation of suspicious densities. Med. Phys 28: 258-266.

29. Otsu N (1979) A treshold selection metod for gray-level histograms. IEEE Trans Syst Man Cyber 9: 62-66.

30. SoilleP (1999) Morphological Image Analysis: Principles and Applications. Springer: 164-165

31. Di Leo G, Fabbrocini G, Paolillo A, Rescigno O, Sommella P (2009) Towards an Automatic Diagnosis System for Skin Lesions: Estimation of Blue-Whitish Veil and Regression Structures. In: Proceedings of the IEEE $6^{\text {th }}$ International Multi-Conference on System, Signals and Devices (SSD '09), Djerba.

32. Vladimir V, Vassili S, AllaA (2001) A Survey on Pixel-Based Skin Colo Detection Techniques. Graphics and Media Laboratory, Moscow state university, Moscow, Russia.

33. Chien C, Tseng DC (2011) Color image enhancement with exact HSI colo model. International journal of Innovative computing, Information and control.

34. Tasoulis SK, Doukas CN, Maglogiannis I, Plagianakos VP (2010) Classification of Dermatological Images Using Advanced Clustering Techniques. 32nd Annual International Conference of the IEEE EMBS Buenos Aires.

35. Cula OG, Dana KJ, Murphy FP, Rao B K (2004) Skin Texture Modeling International Journal of Computer Vision IJVC 62: 97-119.

36. Gonzalez RC, Woods RE, Eddins SL (2003) Digital Image Processing Using MATLAB. Gatesmark Publishing, New Jersey, Prentice Hal.

37. http://www.mathworks.com/help/index.html

38. Markowski CA, Edward M (1990) Conditions for the Effectiveness of a Preliminary Test of Variance. The American Statistician 44: 22-326.

39. Sawilowsky S (2002) Fermat, Schubert, Einstein, and Behrens-Fisher: The Probable Difference Between Two Means When $\sigma_{1}^{2} \neq \sigma_{2}^{2}$. Journal of Modern Applied Statistical Methods 1: 461-472.

40. McDonald JH (2008) Handbook of Biological Statistics. Sparky House Publishing, Baltimore.

41. Golub T, Slonim D, Tamaya P, Huard C, Gaasenbeek M, et al. (1999) Molecular classification of cancer: Class discovery and class prediction by gene expression monitoring. Science 286: 531-537.

42. Sordo M (2002) Introduction to Neural Networks in Healthcare. Open Clinical.

43. Duda RO, Hart PE, Stork DG (2001) Pattern Classification. Wiley $2^{\text {nd }}$ eds.

44. Scholkopf B, Kah-Kay S, Burges CJ, Girosi F, Niyogi P, et al. (1997) Comparing support vector machines with Gaussian kernels to radial basis function classifiers. IEEE trans Signal Processing 45: 2758-2765.

45. Pontil M, Verri A (1998) Support vector machines for 3-D object recognition IEEE Trans. pattern anal. Machine Intel 20: 637-646.

46. Wan V, Campbell WM (2000) Support vector machines for speaker verification and identification. IEEE Workshop Neural Networks for Signal Processing: 775784

47. Osuna E, FreundR, Girosi F, "c," in Proc. (1997) Computer Vision and Pattern Recognition. Puerto Rico 130-136.

48. Joachims T (1999) Transductive inference for text classification using support vector machines. Published at the Int. Conf. Machine Learning, Slovenia.

49. Schölkopf B, Smola A J (2002) Learning with Kernels. MIT Press.

50. Hastie T, Tibshirani R, Friedman J (2003) The Elements of Statistical Learning Second Edition, New York, Springer Verlag.

51. Cristianini N, Shawe-Taylor J (2000) Support Vector Machines. Cambridge, U.K, Cambridge Univ. Press.

52. Nishikawa RM, Giger ML, Doi K, Vyborny CJ, Schmidt RA (1995) Computer aided detection of clustered microcalcifications in digital mammograms. Med BiolEng Comp 33: 174- 178.

53. TheodoridisS, Koutroumbas S (1999) Pattern Recognition. Academic Press, San Diego. 